\title{
Ein renaissancezeitlicher Bauplan des Heidelberger Schlosses in der WLB
}

Im Rahmen eines Forschungsprojektes zur Inventarisierung und wissenschaftlichen Bearbeitung mittelalterlicher Architekturzeichnungen im deutschsprachigen Raum beschäftigte sich der Verfasser dieses Aufsatzes u.a. mit der Sammlung des württembergischen Generals und Kriegsministers Ferdinand Friedrich von Nicolai (1730-1814) in der Württembergischen Landesbibliothek, einem mehrere Tausend Einzelzeichnungen umfassenden Ingenieurnachlass. ${ }^{1}$ Bei dieser Gelegenheit fanden sich unter sehr zahlreichen handgezeichneten Bauplänen einige interessante, bislang noch unbekannte spätgotische Kapellenentwürfe, deren Besprechung in der abschließenden Publikation des oben genannten Projektes ${ }^{2}$ erfolgen wird. Als besonders spektakulär entpuppte sich bei der Recherche nach gotischen Baurissen jedoch ein anderer Fund. Mit dem in Abb. 2 reproduzierten Bauriss besitzt die Württembergische Landesbibliothek eine renaissancezeitliche Architekturzeichnung, welche die Umplanung des Heidelberger Schlosses unmittelbar vor dem Dreißigjährigen Krieg in einem besonders interessanten Teilbereich dokumentiert. Es handelt sich um einen großformatigen Aufriss zum Nordostturm des Schlosses, dem sogenannten Glockenturm, der noch heute - wenn auch in ruinöser Gestalt den berühmten Nordprospekt des Schlosses hoch über der Stadt maßgeblich charakterisiert ${ }^{3}$ (Abb. 1).

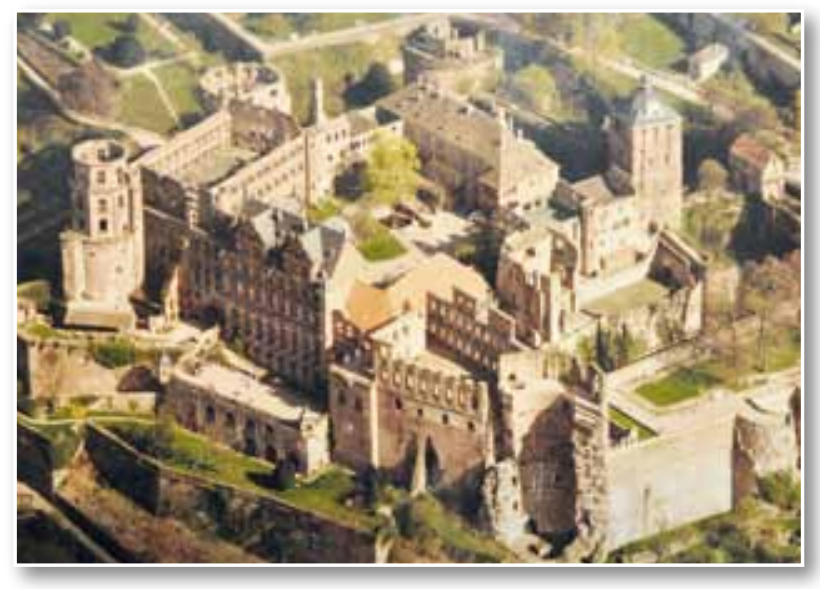

Abb. 1: Schloss Heidelberg, Luftbildaufnahme von Nordwesten, Fotosammlung Schlossbaudirektion Heidelberg.

(1) Zu Ferdinand Friedrich von Nicolai siehe Hohrath u.a. 1990.

(2) Das Inventarwerk über die mittelalterlichen Baurisse der Rheinlande erscheint im April 2013 im Müry Salzmann Verlag Salzburg. Der genaue Titel lautet: Johann Josef Böker, Anne-Christine Brehm, Julian Hanschke, Jean-Sébastien Sauvé: Architektur der Gotik. Rheinlande. Salzburg 2013.

(3) Inv. Nr. Nic.3, BI. 58r.

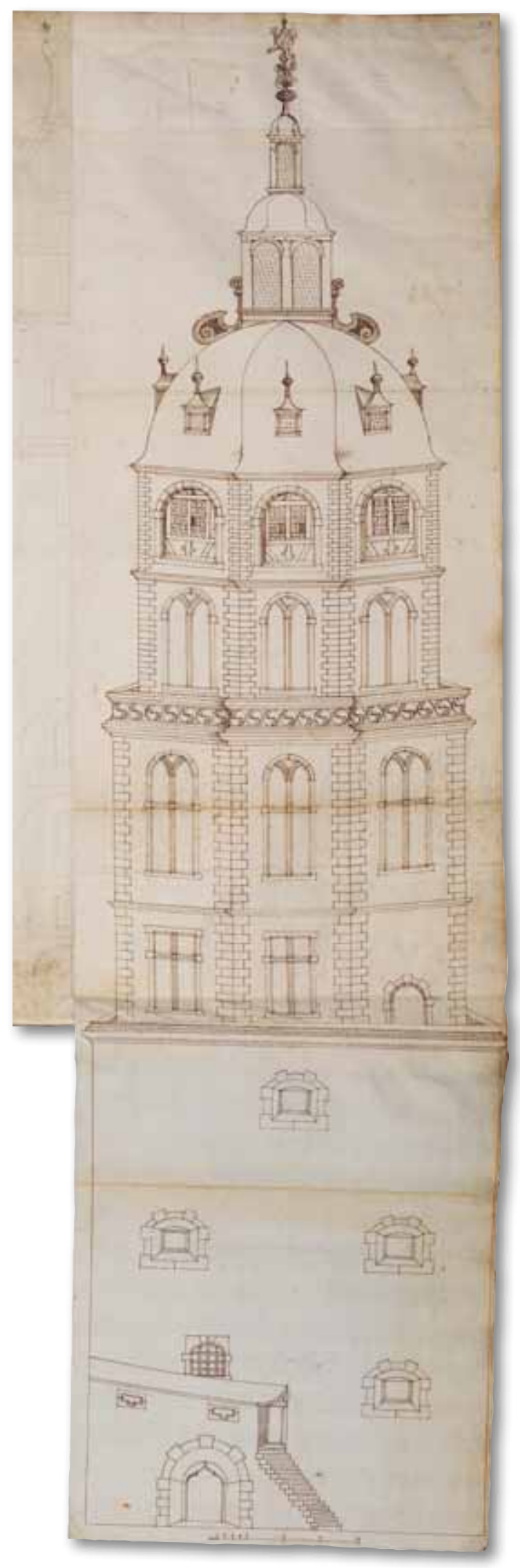

Abb. 2: Aufrisszeichnung zum Glockenturm des Heidelberger Schlosses, um 1608, Sammlung Nicolai, Württembergische Landesbibliothek Stuttgart (Signatur: Nic.3, BI. 58r). 
Die Auffindung dieses Planes ist dabei zweifelsohne als Sensation zu werten, zumal es bislang so schien, als hätten sich außer der Giebelskizze des Ottheinrichsbaues im Wetzlarer Skizzenbuch ${ }^{4}$ keinerlei originale Bauzeichnungen dieses bedeutenden Schlossbaues erhalten. Mit dem hier vorgestellten Bauriss liegt der Forschung nunmehr jedoch - entgegen allen Erwartungen - ein originales planerisches Zeugnis vor, das es erlaubt, die Baugeschichte des nordöstlichen Schlossbereichs weitaus präziser nachzuvollziehen als es bislang der Fall gewesen ist. Im Folgenden seien die Besonderheiten dieses Planes im Zusammenhang mit der Baugeschichte des Glockenturmes erläutert.

\section{Beschreibung des Planes}

Der Bauriss präsentiert den Turm freigestellt von Anbauten, welche sich mit nur geringem Abstand an den Turm anschließen und den Blick auf den Turmunterbau verdecken. Während die unteren Teile der Zeichnung eine Bestandsaufnahme darstellen, sind die obersten beiden Geschosse - wie nachfolgend dargelegt - als Umplanungen zu interpretieren. Um den Turm vom Unterbau bis zum Dach vollständig abbilden zu können, wählte der Planverfasser für seine Bauaufnahme die Ansicht von dem engen Hof hinter dem unter Pfalzgraf Ludwig V. errichteten Zeughaus.

Das untere Drittel der Zeichnung nimmt der zylinderförmige Turmunterbau mit seinen trichterförmig in das Mauerwerk eingelassenen Schießscharten ein. Ausgelassen sind Anbauten an den Turm wie die nördliche fünfeckige Bastionierung mit dem darüber gelegenen spätgotischen Belvedere und der seitliche, nahe des Gläsernen Saalbaus gelegene Treppenturm. Unten links erfasste der Zeichner die lediglich in ihrer Fundamentierung noch erhaltene Treppe zu dem ehemals gedeckten äußeren Umgang, welcher den Turmunterbau wehrhaft verstärkte. Die kielbogigen Tür- und Fenstergestelle unmittelbar ober- und unterhalb des Umganges sind heute nicht mehr erhalten. Sie wurden - wie ein Vergleich mit der Bauaufnahme von Koch/Seitz (Abb. 3, 4) aufzeigt - bei einem späteren Umbau des Turmes durch ein segmentbogiges Portal und ein rechteckiges Fenstergewände ersetzt. Auch die Schießscharten des Umganges lassen sich heute nicht mehr feststellen, da der Umgang bis

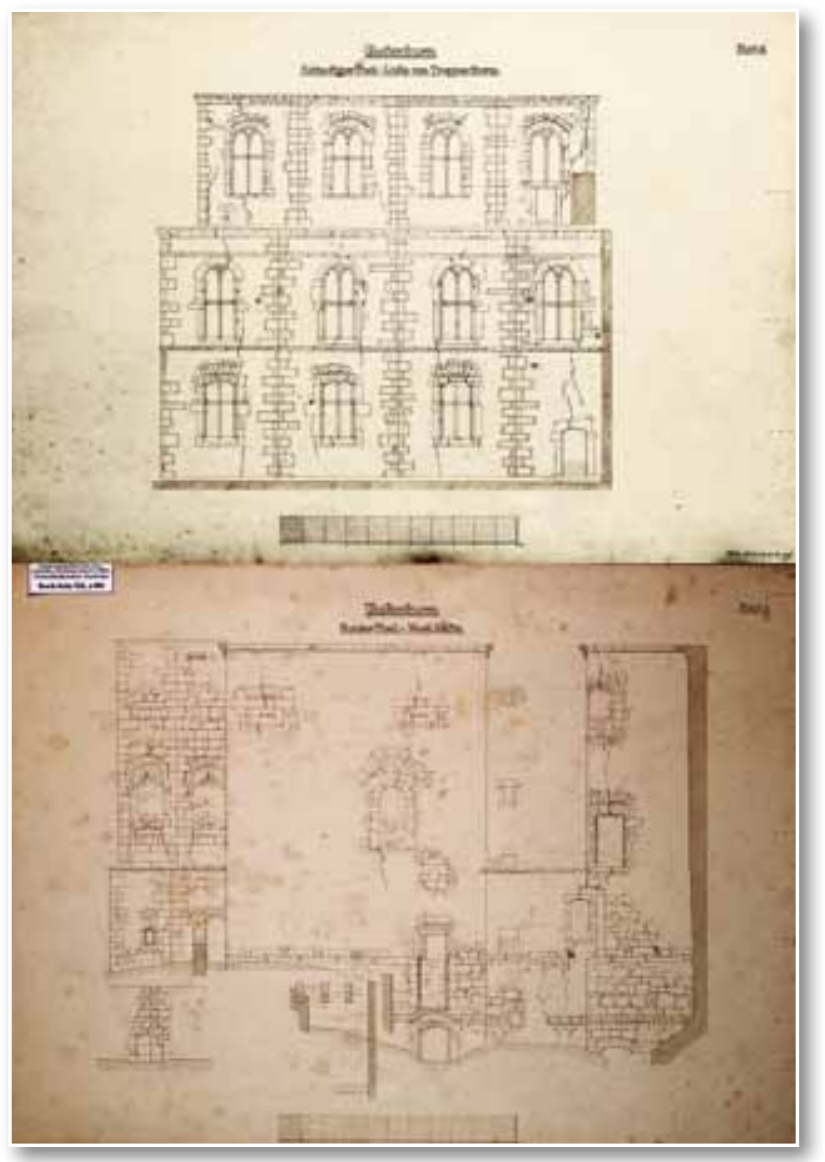

Abb. 3: Wandabwicklungen zum Glockenturm des Heidelberger Schlosses, Bauaufnahmen von Julius Koch und Fritz Seitz, vor 1891, Foto: Generallandesarchiv Karlsruhe.

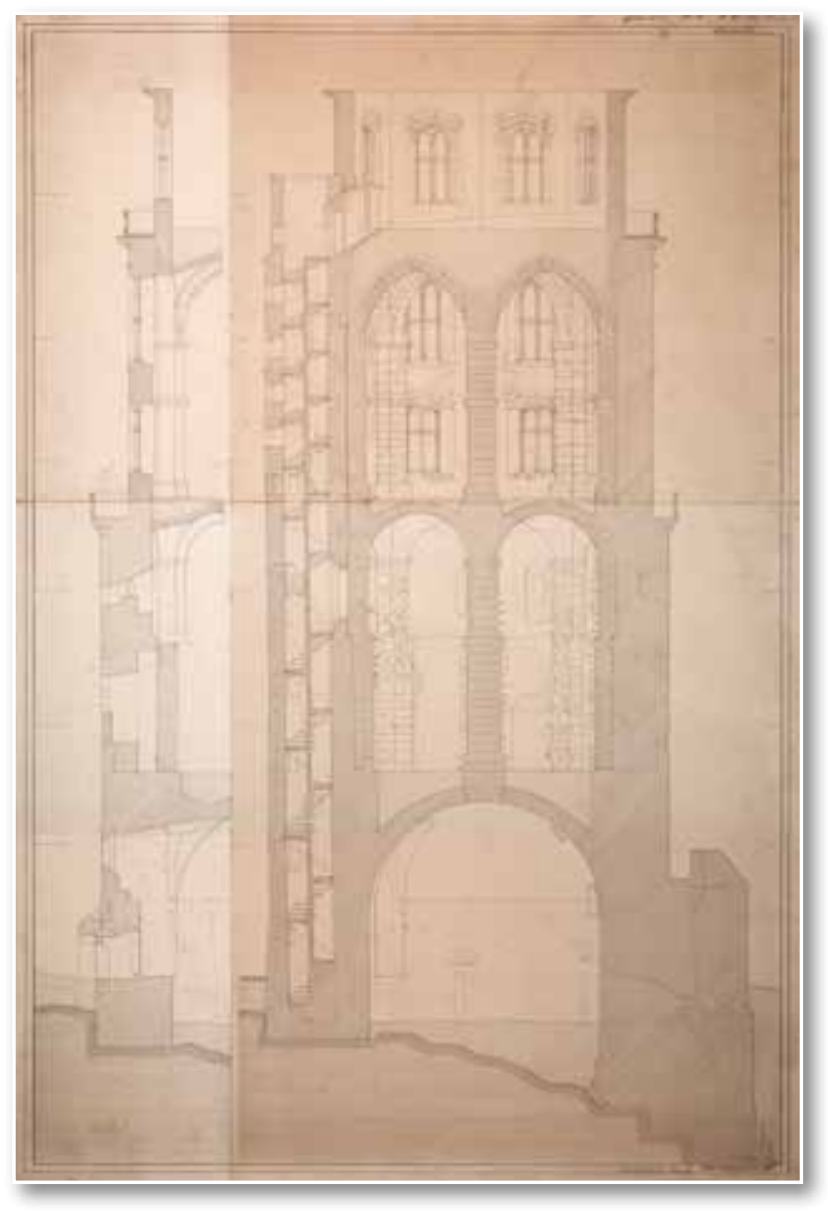

Abb. 4: Schnitt durch den Glockenturm, Bauaufnahme von Julius Koch und Fritz Seitz, vor 1891, Foto: Generallandesarchiv Karlsruhe. 
auf die Reste seines Unterbaus und einige noch vorhandene Konsolsteine während des Pfälzischen Erbfolgekrieges 1689/1693 zerstört bzw. abgetragen wurde.

Oberhalb der glatt verputzten Flächen des Unterbaus gibt die Zeichnung das umlaufende kräftige Gesims wieder, das den massiven Turmunterbau nach oben beschließt. Ein wenig irritierend wirken die im Zusammenhang mit dem Turmunterbau festzustellenden Projektionswechsel, welche den modernen Betrachter an vergleichbare Phänomene in der spätgotischen Zeichenmanier erinnern, und offenbar dazu dienten, einzelne Teile des Turmes in ihrer räumlichen Wirkung zu veranschaulichen. Als Projektionswechsel sind zu lesen: das leicht kurvierte Dach des Umganges als auch das obere Gesims, das vorn noch in strenger Parallelprojektion erscheint, dagegen an den Flanken des oktogonalen Turmaufsatzes etwas missverständlich in die dritte Dimension wechselt.

Das Hauptaugenmerk des Planverfassers galt jedoch nicht der Wiedergabe des Turmunterbaus, vielmehr war inm daran gelegen, die vier, durch profilierte Gurte gegeneinander abgesetzten Obergeschosse darzustellen. Letztere sind im Grundriss oktogonal angelegt und verjüngen sich ab dem zweiten Obergeschoss, so dass Platz für einen weiteren Umgang entsteht. Alle Kanten sind dem heutigen Baubestand entsprechend mit gequaderten Ecken versehen. Über den unteren Kreuzstockfenstern folgen die noch heute erhaltenen Rundbogenfenster, deren innere Fensterbahnen unter dem übergreifenden Rundbogen spitzbogig schlieBen. Eine Abweichung vom heutigen Baubestand stellt lediglich die Brüstung dar, welche anstelle des heutigen einfachen Mauerstreifens mit einer Folge von gotischen Zweischneußen geschmückt ist. Über dem ähnlich gestalteten zweiten Obergeschoss gibt die Zeichnung einen heute nicht mehr erhaltenen Turmaufsatz an, der die Eckquaderung der unteren Geschosse fortführt und von einem hoch aufragenden geschweiften Dach überdeckt ist. Anstelle der zweigeteilten Rundbogenfenster der darunterliegenden Geschosse öffnet sich das oberste Turmstockwerk in großen profilierten Rundbogenarkaden, welche bis auf ein kleines Fensterpaar pro Achse mit schmückendem Fachwerk verfüllt sind. Als Turmabschluss präsentiert die Zeichnung eine oktogonale, mit zwei übereinandergestapelten Laternen geschmückte "Welsche Haube". Unklar wirkt in diesem Abschnitt der Zeichnung auf den ersten Blick die Geometrie der beiden Laternen, doch dürften die beiden aufeinanderfolgenden Turmaufsätze - trotz des oktogonalen Unterbaus - tatsächlich als quadratische Baukörper mit geschweiften Hauben gedacht gewesen sein. Als verbindendes Motiv zwischen den Turmaufsätzen und dem Hauptdach des Turmes fügte der Zeichner zwei kunstvoll geformte seitliche Voluten ein.

\section{Historische Ansichten}

Um die Entstehungszeit des Planes zu bestimmen, ist es erforderlich, die Baugeschichte des Turmes anhand der erhaltenen historischen Ansichten in ihren wesentlichen Etappen nachzuzeichnen. Die früheste topographisch getreue Bildquelle des

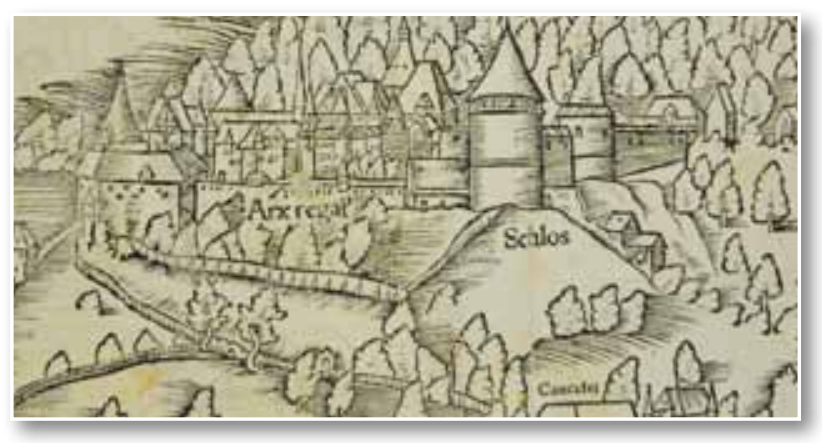

Abb. 5: Sebastian Münster, Ansicht des Schlosses auf dem Heidelbergpanorama der Cosmographia, Holzschnitt um 1549.

Heidelberger Schlosses liefert die große, vor 1549 entstandene Heidelberg-Ansicht des berühmten Theologen und Kosmographen Sebastian Münster (Abb. 5). Auf dem großformatigen Holzschnitt ist der Turm mit einem steilen kegelförmigen Dach über dem heutigen Turmunterbau dargestellt. Die oktogonalen Obergeschosse bestanden zu jener Zeit noch nicht.

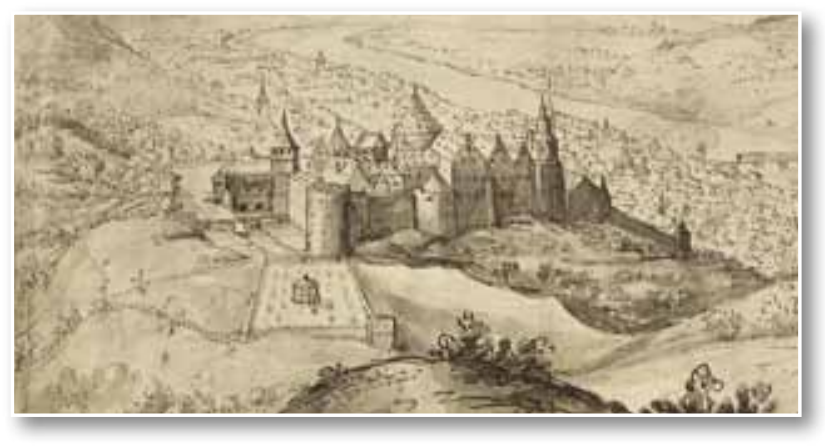

Abb. 6: Unbekannter Künstler, Ansicht des Schlosses von Südosten, um 1590, Kurpfälzisches Skizzenbuch, Staatsgalerie Stuttgart. 
Auf der Ansicht von Jan Breughel (1568-1625) im New Yorker Metropolitan Museum of Art und den um 1580-1590 entstandenen Bildern des Kurpfälzischen Skizzenbuches in der Staatsgalerie Stuttgart $^{5}$ (Abb. 6) erscheint der Turm dagegen bereits mit der nachträglichen Aufstockung. Der oberste Turmaufsatz war zu diesem Zeitpunkt kuppelartig geformt und mit einer gedrungenen Laterne versehen.

Eine erneute Veränderung erfuhr das Bauwerk in der Zeit zwischen 1603 und 1619. Während der Turm auf der kolorierten Schlossansicht des Thesaurus Picturarum in seinen Details noch ziemlich exakt der Ansicht des Jan Breughel gleicht, erkennen wir auf dem Gemälde von Jacques Fouquières (1580/1591-1659) im Kurpfälzischen Museum

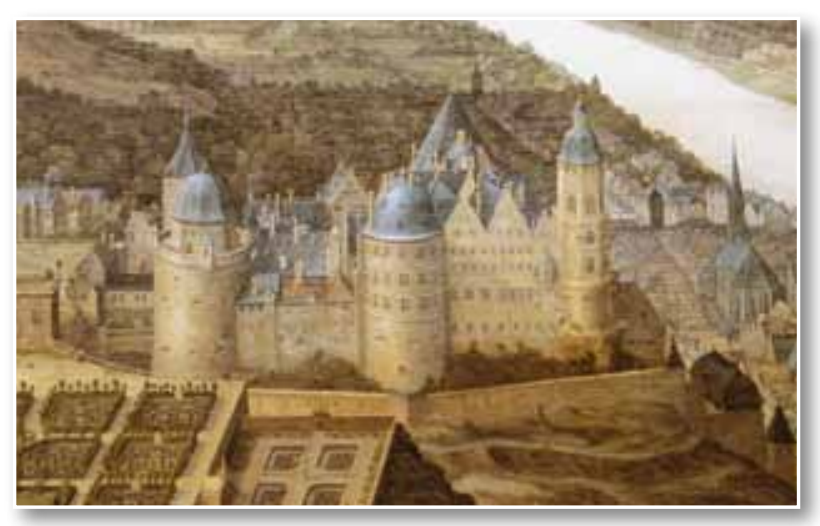

Abb. 7: Jacques Fouquières, Ansicht des Schlosses von Osten, um 1619, Kurpfälzisches Museum Heidelberg.

Heidelberg (Abb. 7) das erst zu dieser Zeit erbaute (heutige) dritte Oktogongeschoss, das sich in seinen Formen an die untere Gestaltung anlehnt, jedoch nach Ausweis der besprochenen Ansichten einer wesentlich späteren Bauphase angehören muss. Oberhalb dieses neuen Turmaufsatzes folgte nach Fouquières ein weiteres, an den acht Seiten mit jeweils einem Doppelfenster ausgestattetes Stockwerk mit geschweifter Haube und oktogonaler Laterne.

Die Übereinstimmungen der Darstellung des Turmes in dem Gemälde mit dem Bauplan (Abb. 2) lassen darauf schließen, dass derselbe wohl eine Planungsstufe zu genau diesem Bauabschnitt darstellt und demnach in der Zeit zwischen 1603 (Ansicht im Thesaurus Picturarum des Marcus zum Lamm, Hessische Landes- und Hochschulbibliothek Darmstadt, Hs. 1971) und 1619 (Ansicht von Jacques Fouquières) entstanden ist. Eine Präzisierung dieser Datierung gelingt über eine archivalisch überlieferte Nachricht vom April 1608, wonach "ein nach allen Seiten offener Aufsatz" zur Aufbewahrung der Glocke durch einen gewölbten steinernen ersetzt wurde. ${ }^{6}$

Offensichtlich wurde der Turmaufsatz jedoch wesentlich einfacher ausgeführt als zunächst beabsichtigt. So verzichtete man bei der Bauausführung auf die ursprünglich angedachte doppelte Laternenbekrönung, die großen Rundbögen mit den Fachwerkfüllungen und den seitlichen Volutenschmuck.

Die anhand der historischen Ansichten zweifelsfreie Datierung des Planes in das beginnende 17. Jahrhundert lässt vermuten, dass der Umbau des Turmes mit der Errichtung des Altans (nach 1605) und des Friedrichsbaues ${ }^{7}$ (1601-1605) einherging. Hierauf deutet zumindest der nachfolgende Formenvergleich zwischen den Details der Zeichnung und der Architektur des Altangebäudes und des Friedrichsbaues: Eine ins Auge fallende Gemeinsamkeit zwischen der Zeichnung und dem Altan stellen die großen Rundbögen des obersten Geschosses dar, welche mit den großen Öffnungen der Altanhalle korrespondieren. Darüber hinaus lässt sich auch die zweiachsige geschweifte Laterne formal mit einem Bauteil des Altans kombinieren. Es handelt sich um die Eckpavillons auf der Altanplattform, welche jeweils mit einer

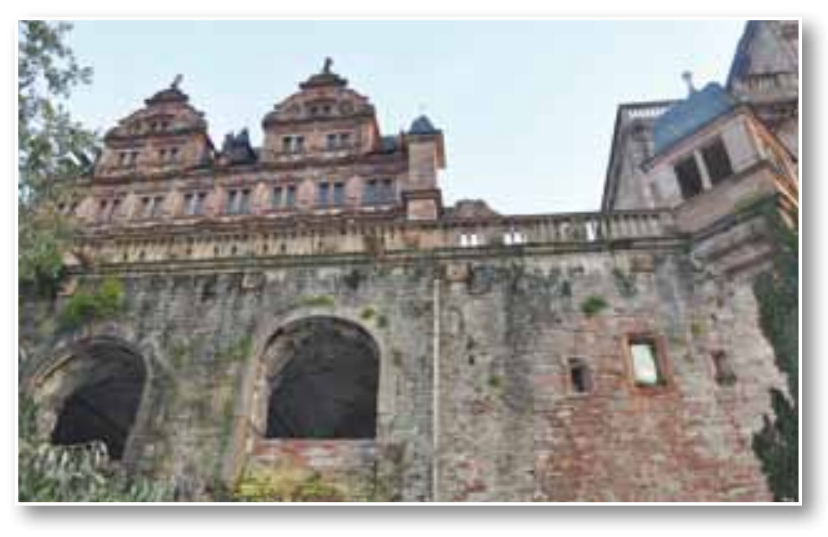

Abb. 8: Nordansicht des Altangebäudes, Foto: Julian Hanschke.

zweiachsigen Frontseite versehen sind und ein geschweiftes Dach tragen (Abb. 8). Einen weiteren gestalterischen Zusammenhang zwischen beiden

(5) Siehe hierzu Hubach 1996. Die Zeichnungen befinden sich in der Staatsgalerie Stuttgart, Inv. Nr. C 95.

(6) Oechelhaeuser 1913, S. 436. Pithopoeus (Annales Academia Heidelbergensis fol. 81b). Zu Pithopoeus siehe auch Rosenberg 1882, S. 156, Anmerkung 2. Neues Archiv für die Geschichte der Stadt Heidelberg und der Kurpfalz, S. 114, Anmerkung.

(7) Oechelhaeuser 1913, S. $471 \mathrm{f}$. 


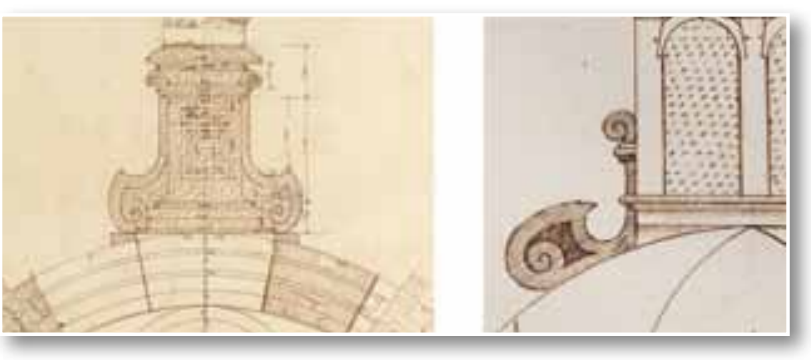

Abb. 9: Links: Voluten an den Giebelbekrönungen des Friedrichsbaues nach der Bauaufnahme von Julius Koch und Fritz Seitz,

rechts: Ausschnitt aus Abb. 2.

Bauten bietet der seitliche Volutenschmuck an der geschweiften Haube. Seine Formgebung ähnelt den Voluten an den Pfeilern der Altanbrüstung und entspricht darüber hinaus fast wörtlich den Voluten seitlich der Figurensockeln auf den Giebeln des Friedrichsbaues (Abb. 9).

Weitere formale Verbindungen mit der Heidelberger Schlossarchitektur um 1600-1620 ergeben sich anhand eines Vergleichs der Zeichnung mit den Architekturentwürfen des Wetzlarer Skizzenbuches, das - bislang ungesehen - u.a. einige Detailzeichnungen der Altanbrüstung enthält. Daneben findet sich auf Tafel 74 des Wetzlarer Skizzenbuches die Darstellung eines Obelisken mit der Jahreszahl 1617, welcher mit gleichartigen Voluten entsprechend der Glockenturm-Zeichnung geschmückt ist.

\section{Das Heidelberger Schloss - ein Beispiel für nachgotische Architektur}

Die für den vorliegenden Bauplan charakteristische Verbindung gotischer und renaissancezeitlicher Formen (Fischblasenmaßwerke der Brüstung und geschweifte Haube) mag zunächst irritieren, ist jedoch als ein Stilmerkmal der Zeit um 1600 gängig. Prominente Beispiele für die Architektur der sogenannten Nachgotik in der ersten Hälfte des 17. Jahrhunderts stellen unter anderem die Kirchen St. Mariä Himmelfahrt in Köln oder die Jesuitenkirche in Molsheim dar. Dabei müssen jedoch keine Vergleichsbeispiele weit außerhalb gesucht werden, denn das Heidelberger Schloss selbst ist in wesentlichen Teilen als treffendes Beispiel für das Phänomen „nachgotischer" Architektur zu interpretieren, auch wenn dieser interessante Aspekt in der bisherigen Heidelberg-Literatur kaum eine Würdigung erfahren hat. Das wichtigste Werk der Nachgotik auf dem Heidelberger Schloss ist unzweifelhaft die Kapelle des Friedrichsbaus. Mit ihren sternengewölbten Mittelschiffjochen zitiert dieselbe Gewölbefiguren wie sie am Bibliotheksbau des Heidelberger Schlosses, der Heiliggeistkirche und der Peterskiche etwa 100 Jahre früher geschaffen wurden. Ferner ist als nachgotische Architekturform das Motiv des Kreuzstockfensters aufzufassen, das sowohl am Ottheinrichsbau, als auch am Dicken Turm und Glockenturm Anwendung fand. Auch der Altan mit seinen kreuzrippengewölbten Jochen am unteren Eingang und schließlich der Fassbau des Pfalzgrafen Johann Kasimir mit seinen gotischen Maßwerkfenstern und dem komplizierten figurierten Gewölbe der sogenannten Fassbaukapelle stellen qualitätvolle Zeugnisse der Nachgotik dar und tradieren die Bauformen der spätgotischen Architektur des Schlosses in das Zeitalter der Renaissance und des Frühbarocks.

\section{Baubeschreibung des Turmes}

Wie die meisten Bauten des Heidelberger Schlosses wurde auch der Glockenturm im Vorfeld der Restaurierungsarbeiten des ausgehenden 19. Jahrhunderts durch die Architekten Julius Koch und Fritz Seitz detailgenau vermessen. Es handelt sich um einen sehr umfangreichen Plansatz von über 30 Zeichnungen, welche den Turm als Fassadenabwicklung, Schnitte, Grundrisse und Baudetails eingehend dokumentieren (Abb. 3, 4).

Von besonderem Interesse ist zunächst der zylinderförmige Unterbau des Turmes mit seinem leicht geböschten Sockel, der eine ganze Anzahl baugeschichtlich relevanter Befunde enthält. Im nachfolgenden Abschnitt seien die wichtigsten dieser Befunde näher beschrieben und einer Bewertung zugeführt.

Als Hinweis auf einen relativ niedrigen, nur etwa 16 Meter hohen ursprünglichen Turmbau interpretierten Koch und Seitz ein Ringankersystem aus ineinandergreifenden u-förmigen Werksteinen knapp oberhalb des geböschten Turmunterbaus. „Es musste [durch den Einbau dieses Ankers] dem Seitenschub widerstanden werden, weil das belastende Mauerwerk über dem Widerlager des Gewölbes eine geringe Höhe hatte. "8

An weiteren Besonderheiten sind der kleine Treppenturm zur Erschließung der Turmgeschosse an der Südwestseite nahe dem Gläsernen Saalbau 
und die drei Miniaturbastionen zu nennen, welche sich im nordöstlichen Bereich mit dem Turmunterbau verbinden (Abb. 3, 4). Die genannten Bastionen lassen sich als kleine, massiv gemauerte fünfeckige Vorsprünge beschreiben; sie dienten offenkundig dazu, den auf Konsolsteinen vorkragenden Wehrgang zu tragen. Die Bastionen selbst besaßen nach den Ansichten des 17. Jahrhunderts ursprünglich pyramidal gemauerte Aufsätze, während das Dach des Wehrganges, wie der hier besprochene Bauplan belegt, wie üblich aus Holz gezimmert war. Nicht mehr erhalten ist die ebenfalls im Plan festgehaltene Treppe zum Wehrgang, deren früherer Standort jedoch noch anhand vorhandener Anschlussspuren nachgewiesen werden kann.

Unter den zahlreichen in Werkstein ausgeführten Fenster- und Türgewänden sind zu erwähnen: zwei übereinanderliegende Portale mit geschweiften spätgotischen Stürzen nahe der Anschlussstelle an den Gläsernen Saalbau, ein gleichartiges Portal in dem unterirdischen Verbindungsgang zwischen Gläsernem Saalbau und Glockenturm und drei dreiteilige Fenstergewände mit erhöhter Mittelöffnung und kielbogigem Blendmaßwerkschmuck an der Ost- und Westseite des Turmes. Derselben spätgotischen Bauphase des Turmes ist ferner der Aufbau auf der vorderen nördlichen fünfeckigen Bastion zuzurechnen. Es handelt sich um ein turmartiges Gebilde, das oberhalb eines gotischen Kaffgesimses vier größtenteils vermauerte kielbogige Fensteröffnungen enthält, die wahrscheinlich keine steinerne Binnengliederung (Maßwerk) besaßen. Ferner bewahrt der gleiche Anbau zwei kleinere Fensterchen ebenfalls mit Kielbogenblendmaßwerk im Bereich unterhalb des genannten Kaffgesimses auf der Höhe des äußeren Umganges. In späterer Zeit erhielt der Turmunterbau eine ganze Anzahl unregelmäßig verteilter trichterförmiger Schießscharten mit segmentbogig gemauerten Stürzen.

Im Inneren bewahrt der zweigeschossige Unterbau einen Kellerraum mit halbkugelförmigem Gewölbe und Zugang in den Keller des Gläsernen Saalbaus wie auch in den Hof des Zeughauses.

Das hohe Geschoss über dem Keller besaß ursprünglich, wie Koch und Seitz feststellten, ein figuriertes spätgotisches Gewölbe, von dem Ende des 19. Jahrhunderts noch geringfügige Spuren der Konsolen und Schildbögen festzustellen waren. Dasselbe wurde zum Zeitpunkt der Erbauung der Obergeschosse (um 1550) abgebrochen und im frühen 17. Jahrhundert durch eine höchst waghalsige Konstruktion - ein Gewölbe mit Mittelstütze, welche auf der Halbkugelschale des Kellers gegründet ist, ersetzt (Abb. 4). Zum gleichen Zweck erhielt der Raum aus Werksteinquadern gemauerte Wandvorlagen mit Kämpfergesimsen, welche als Auflager für das mit einem Stichkappensystem versehene Gewölbe verwendet wurden.

\section{Bisherige Rekonstruktionsversuche zu den Bauphasen des Unterbaus}

Nach Koch/Seitz wurde der Turm bereits im späten 15. Jahrhundert einschließlich der bastionsartigen Vorsprünge errichtet, besaß jedoch mutmaßlich zunächst nur eine geringe Höhe (bis knapp oberhalb des halbkugelförmigen Gewölbes), welche mit dem gleichartigen Mauerwerk des Burggeviertes korrespondierte. Als oberen Turmabschluss vermuteten Koch und Seitz eine zinnenbekrönte Plattform und ein dahintergelegenes Kegeldach. ${ }^{9}$ Gleichzeitig wurde angenommen, dass die Bastionen zunächst allesamt dieselbe Höhe wie der eigentliche Turm aufwiesen. Für diese These konnten Koch/Seitz jedoch nur wenige stichhaltige Argumente nennen. Problematisch erscheint insbesondere, die Existenz des Ringankers am Fuße des Turmzylinders als Argument für die vermutete niedrige Höhe des Turmes heranzuziehen, ${ }^{10} \mathrm{da}$ auch bei einem höheren Gebilde ein Ringankersystem an dieser Stelle zweckdienlich gewesen wäre, um die Substruktionen des Turmes zu verstärken. Darüber hinaus mag den Bastionen lediglich die Aufgabe zugekommen sein, den vorgelagerten Wehrgang zu verstärken, so dass auch hier fraglich ist, ob überhaupt von einer Veränderung gegenüber dem späteren Zustand (vgl. Gemälde von Fouquières) auszugehen ist.

Als zweite Bauphase wurde von Koch/Seitz die Errichtung des ersten Obergeschosses mit dem heute nicht mehr vorhandenen figurierten Gewölbe, den spätgotischen Portalen und den Drillingsfenstern mit Kielbogenblendmaßwerk sowie der Bau eines belvedereähnlichen Gehäuses mit kielbogigen Öffnungen auf der vorderen Bastion 
interpretiert. ${ }^{11}$ Doch widerspricht eine zeitliche Trennung des Obergeschosses vom Unterbau der Tatsache, dass bereits der Unterbau mit gleichartigen spätgotischen Vorhangportalen ausgestattet ist, wie sie auch am oberen Teil des Turmzylinders vorkommen. Da alle Portale störungsfrei mit dem Mauerwerk in Verbindung stehen, besteht kein Grund, einen Umbau zu vermuten; vielmehr ist davon auszugehen, dass der gesamte Turmunterbau nach Ausweis der gleichartigen Portale in einem Zug einschließlich des ehemaligen figurierten Gewölbes und der Drillingsfenster wie auch des Belvederes auf der vorderen Eckbastion errichtet wurde. Bezüglich der ursprünglichen Turmhöhe ist darüber hinaus anzunehmen, dass dieselbe die heutige Oberkante des Turmzylinders bereits erreichte und über dem figurierten Gewölbe, anders als es Koch und Seitz in ihrer Rekonstruktionszeichnung angeben, noch ein niedriges weiteres Geschoss aufsaß. Auch für diese Überlegung sind wiederum die spätgotischen Portale, von denen sich jeweils eines auf jedem Stockwerk in störungsfreiem Mauerverband erhalten hat, als Beweis anzuführen. ${ }^{12}$ Schließlich bleibt noch zu erwähnen, dass sich auch am äußeren Mauerwerk des Turmzylinders keine Anzeichen einer nachträglichen Erhöhung feststellen lassen. Damit spricht alles dafür, dass der Turm in seinem ersten vollendeten Bauzustand so aussah, wie er von Sebastian Münster einschließlich des kleinen benachbarten Treppenturmes auf der Stadtansicht von 1540/50 abgebildet wurde.

Diese These deckt sich überdies mit der schriftlichen Überlieferung zum Heidelberger Schloss. So findet sich in der Schloss-Beschreibung des Biographen Ludwigs V., Hubertus Thomas Leodius, die Bemerkung, dass der Turm unter Ludwig $V$. errichtet und von dessen Bruder Friedrich II. (um die Oktogongeschosse) erhöht wurde. ${ }^{13}$ Nach diesen Ausführungen muss davon ausgegangen werden, dass der Glockenturm zwischen 1508 und 1544, der Amtszeit Ludwigs V., entstanden ist, wobei die Verwendung von Bruchstein anstelle von sauber geschichtetem Quadermauerwerk, wie es für die späteren Bauten Ludwigs $\vee$. charakteristisch ist (Dicker Turm, Stück-

(11) Koch/Seitz 1891, S. 26, 28

(12) Lediglich das Portal, welches das Kellergeschoss mit dem Gläsernen Saalbau mittels eines unterirdischen Ganges verbindet, wirkt wie später eingesetzt. Es handelt sich jedoch ganz offensichtlich um eine moderne Erneuerung nach altem Vorbild. Darüber hinaus sei angemerkt, dass sich das benachbarte, gleichartig gestaltete Treppenturmportal unverändert erhalten hat.

(13) Siehe hierzu Koch/Seitz 1891, S. 21. garten, Torturm), auf eine relativ frühe Zeitstellung in

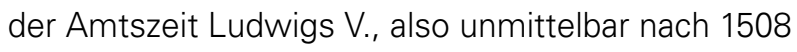
bis ca. 1525, schließen lässt. Als obere Zeitgrenze ist für die Vollendung des Turmes das Jahr $1527 \mathrm{zu}$ nennen. Dies bestätigt der kleine Miniaturholzschnitt Heidelbergs in Sebastian Münsters Calendarium Hebraicum, welches das polygonale Zeughaus einschließlich des dahinter gelegenen Turmes mit nur wenigen Strichen treffend wiedergibt.

Aller Wahrscheinlichkeit nach dürfte der Glockenturm somit gleichzeitig mit dem Bibliotheksbau und der Brunnenhalle erbaut worden sein. Hierfür sprechen zumindest die wenigen Schmuckformen, mit denen diese Gruppe von Bauten ausgezeichnet ist. So hat sich beispielsweise auch an der Nordwand des Soldatenbaus ein Fensterchen mit Blendmaßwerkschmuck, wie es am Glockenturm vorkommt, erhalten, und auch die bereits mehrfach erwähnten Portale des Glockenturmes finden an anderer Stelle ein Pendant. Es handelt sich um das Portal in der Westwand des Gläsernen Saalbaus, das wohl in Zweitverwendung hierher gelangte. Schließlich deuten auch die nur noch in Spuren nachzuweisenden Konsolsteine des figurierten Gewölbes im ersten Obergeschoss des Glockenturmes auf eine enge Formverwandtschaft mit den Kapitellen und Konsolen, die sich am Soldatenbau und am Bibliotheksbau erhalten haben.

Interessanterweise lassen sich für die WerksteinSchmuckformen des Glockenturmes einige weitere Vergleichsbeispiele anführen. So kehrt das Motiv eines Kielbogenfensters ohne Maßwerkfüllung in ganz ähnlicher Form auch am Nordturm des Wormser Domes wieder, dessen Obergeschoss um 1480/90 in spätgotischen Formen erneuert wurde.

Für die charakteristischen Drillingsfenster mit Kielbogenblendmaßwerk finden sich einzelne Parallelfälle in elsässischen Städten. Ein Beispiel stellt das leider undatierte Haus zum Schwan in Colmar (bislang irrig als das Wohnhaus Schongauers interpretiert) dar. Ferner lässt sich als wesentlich reicher durchgestaltetes Beispiel für die Verwendung von Kielbögen in der Profanarchitektur des Spätmittelalters das "Haus zum Walfisch" in Freiburg anführen, welches um 1516 entstand, und somit etwa gleichzeitig mit dem Unterbau des Glockenturmes zu datieren ist. 


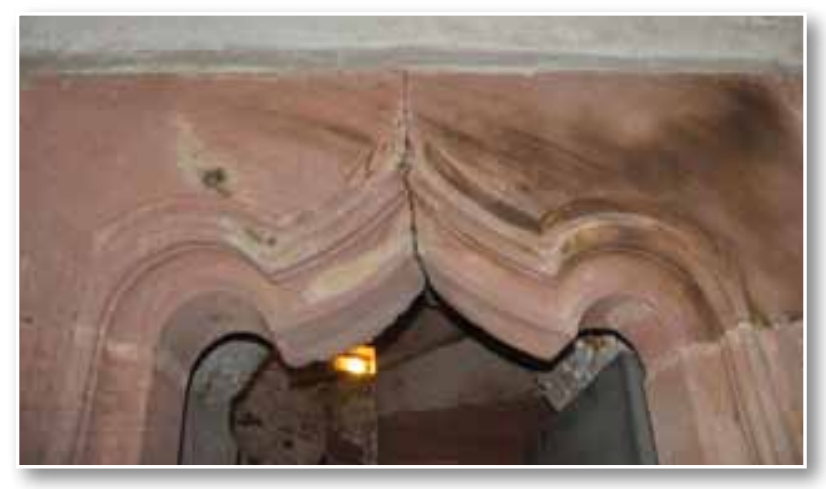

Abb. 10: Spätgotisches Portal im Unterbau des Glockenturmes, Foto: Julian Hanschke.

Auf eine ähnliche Zeitstellung deutet die Portalarchitektur des Glockenturmes hin (Abb. 10). Durchaus verwandte Formen zeigen zwei auf 1483 und 1490 datierte Portale in Konstanz. Doch mag die Art der Portalgestaltung eher mit gleichartigen Gestaltungen an den großen Residenzen verbunden sein. Wie ein Blick auf Abb. 11 verrät,

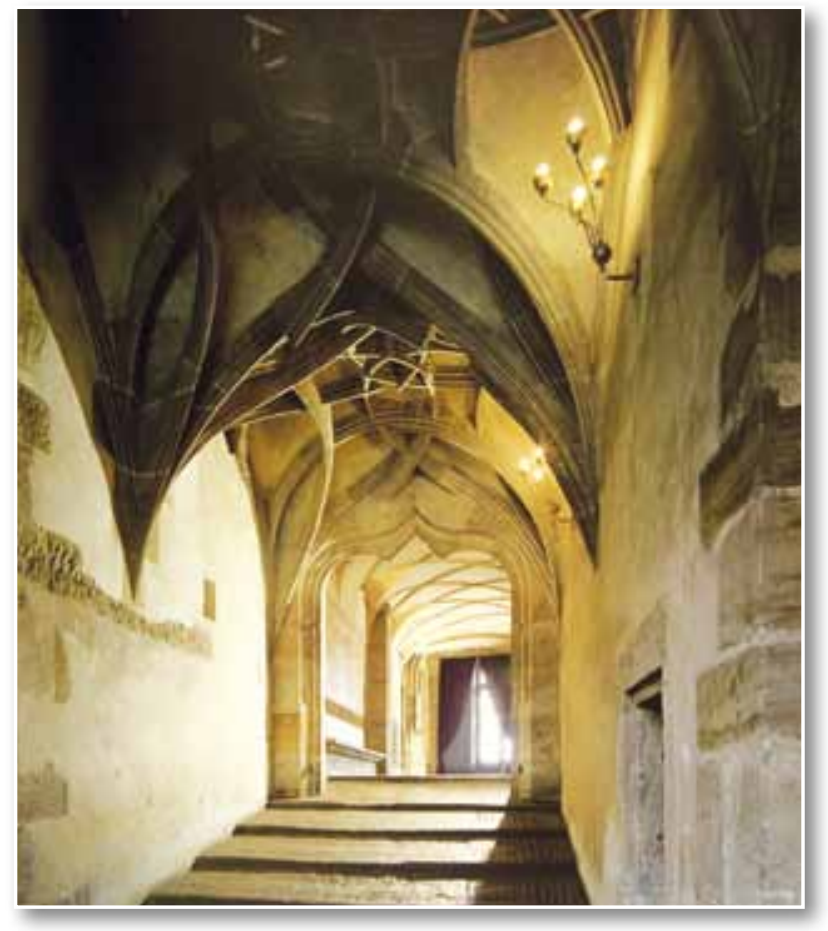

Abb. 11: Reitertreppe des Benedikt Ried im Prager Hradschin, Foto aus Hucek 1992.

könnte sich der Baumeister des Glockenturmes die Portalarchitektur der Reitertreppe im Prager Hradschin (ca. 1490-1502) zum Vorbild genommen haben. Dass sich Pfalzgraf Ludwig V. u.a. an den innovativen Bauten der Prager Burg orientierte, um das Heidelberger Schloss in eine standesgemäße frühneuzeitliche Residenz zu verwandeln, liegt dabei durchaus nahe, zumal Prag in der Zeit um 1500 ein architektonisches Zentrum von europäischem Rang präsentierte. ${ }^{14}$ Sehr wahrscheinlich ist, dass der für den spätgotischen Umbau des Schlosses verantwortliche Werkmeister Lorenz Lechler Prag gekannt hat, und die neuesten Formen, die Benedikt Ried (1454-1534) an der Prager Burg geschaffen hatte (Wladislaw-Saal, Reitertreppe etc.), eingehend studierte. In diesem Zusammenhang ist erwähnenswert, dass Ludwig V. 1509 Wladislaus von Böhmen in Prag besuchte, um sich "seine oberpfälzischen Lehen bestätigen zu lassen". ${ }^{15}$

Neben der Beziehung zur Architektur des Benedikt Ried zeigt die Portalarchitektur des Heidelberger Glockenturmes darüber hinaus eine sehr enge Verwandtschaft zur Architektur des Augsburger Werkmeisters Burkhard Engelberg (1447-1512), der neben dem Ulmer Münsterbaumeister Matthäus Böblinger (um 1450-1505) zu den führenden Architektenpersönlichkeiten seiner Zeit zählte und durch ein recht großes nachweisbares CEuvre auffällt. ${ }^{16}$ Sein Hauptwerk, die Klosterkiche St. Ulrich und Afra in Augsburg, zeichnet sich durch eine mit den Bauten Lorenz Lechlers grundsätzlich übereinstimmende Formensprache aus. Besonders kurios erscheint in diesem Zusammenhang, dass,

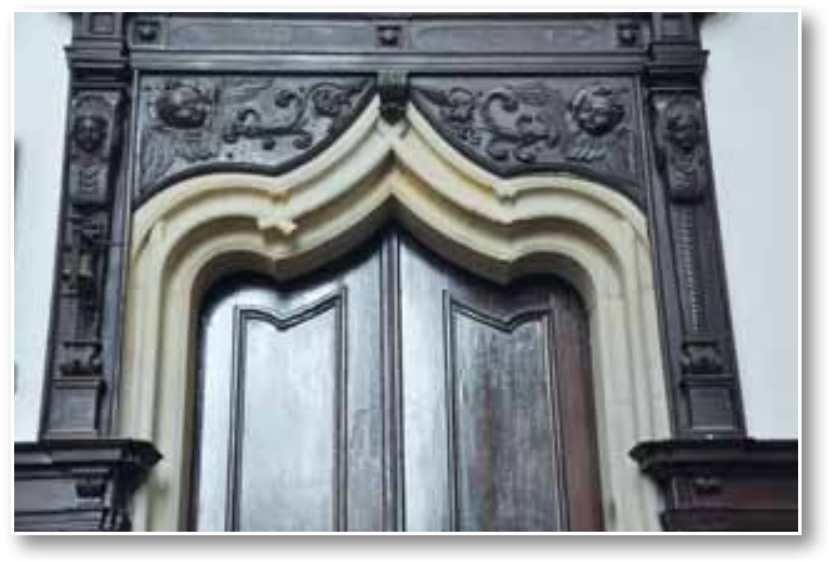

Abb. 12: Portal des Augsburger Werkmeisters Burkhard Engelberg im südlichen Seitenschiff von St. Ulrich und Afra in Augsburg, Foto: Julian Hanschke.

wie Abb. 12 belegt, Burkhard Engelberg ein fast gleichartiges Portal im südlichen Seitenschiff von St. Ulrich und Afra etwa 20 Jahre zuvor geschaffen hat. Die Übereinstimmungen in der Profilierung wie auch in der Formgebung lassen dabei vermuten, dass Lorenz Lechler womöglich einige Lehrjahre in Augsburg bei seinem 15-20 Jahre älteren Kollegen Burkhard Engelberg verbracht hatte und dessen Formengut wenige Jahre später in Heidelberg rezipierte. 


\section{Weitere Bauphasen}

Wann genau die oberen Partien des Turmes errichtet wurden, lässt sich anhand der oben bereits genannten Schriftquelle relativ präzise bestimmen. Nach der Schlossbeschreibung des Leodius entstanden die Obergeschosse des Glockenturmes unter Friedrich II., und zwar unmittelbar vor 1551, dem Datum der von Hubertus Thomas Leodius überlieferten Aufhängung einer Glocke. Diese Datierung verbindet sich problemlos mit den Stilformen, welche die unteren beiden Oktogongeschosse auszeichnen. Um 1549 war der Vorgängerbau des Gläsernen Saalbaues, ein dreigeschossiges Fachwerkhaus, zugunsten des heutigen Gläsernen Saalbaus abgebrochen worden. Mit dem neuen Wohngebäude in der Nordostecke des Schlosshofes dürfte demnach die Erhöhung des Glockenturmes einhergegangen sein. Die verwendeten gotischen Formen, Kreuzstockfenster und spitzbogige Fensterbahnen innerhalb übergreifender Rundbögen, entsprechen den retrospektiven nachgotischen Stilformen der Zeit des mittleren 16. Jahrhunderts. ${ }^{17}$ Nur wenige Jahre später entstand die ebenfalls mit Kreuzstockfenstern ausgestattete Rückfassade des auf 1556 - 1559 datierten Ottheinrichsbaues. togongeschoss des Glockenturmes übereinstimmend Rundbogenfenster mit doppelten inneren Spitzbogenbahnen besaß.

Mit der Bautätigkeit unter Friedrich II. hatte der Glockenturm jedoch noch nicht seine endgültige Gestalt erhalten. Nur wenige Jahre nach der Aufstockung um zwei weitere Oktogongeschosse in der Zeit um 1608 wurde das Bauwerk offenbar während der Belagerung durch die Schweden im Jahre 1633 beschädigt, ${ }^{18}$ denn die Ansichten des späten 17. Jahrhunderts zeigen den Turm wiederum mit einer veränderten Dachgestalt. Anstelle des obersten Oktogongeschosses war in der zweiten Hälfte des 17. Jahrhunderts ein geringfügig kurviertes Zeltdach mit hoch aufragender geschweifter Laterne errichtet worden (Abb. 13). Doch auch dieser Bauzustand sollte nicht allzu lange Bestand haben, denn mit der Zerstörung des Schlosses im Pfälzischen Erbfolgekrieg wurde der Glockenturm, der dem Versuch einer Sprengung standhielt, wiederum seiner Dachwerke beraubt. Während der Wiederaufbaumaßnahmen unter Kurfürst Carl Philipp um das Jahr 1718 erhielt der Turm in Anlehnung an den Bauzustand des 17. Jahrhunderts erneut eine geschweifte Haube, welche

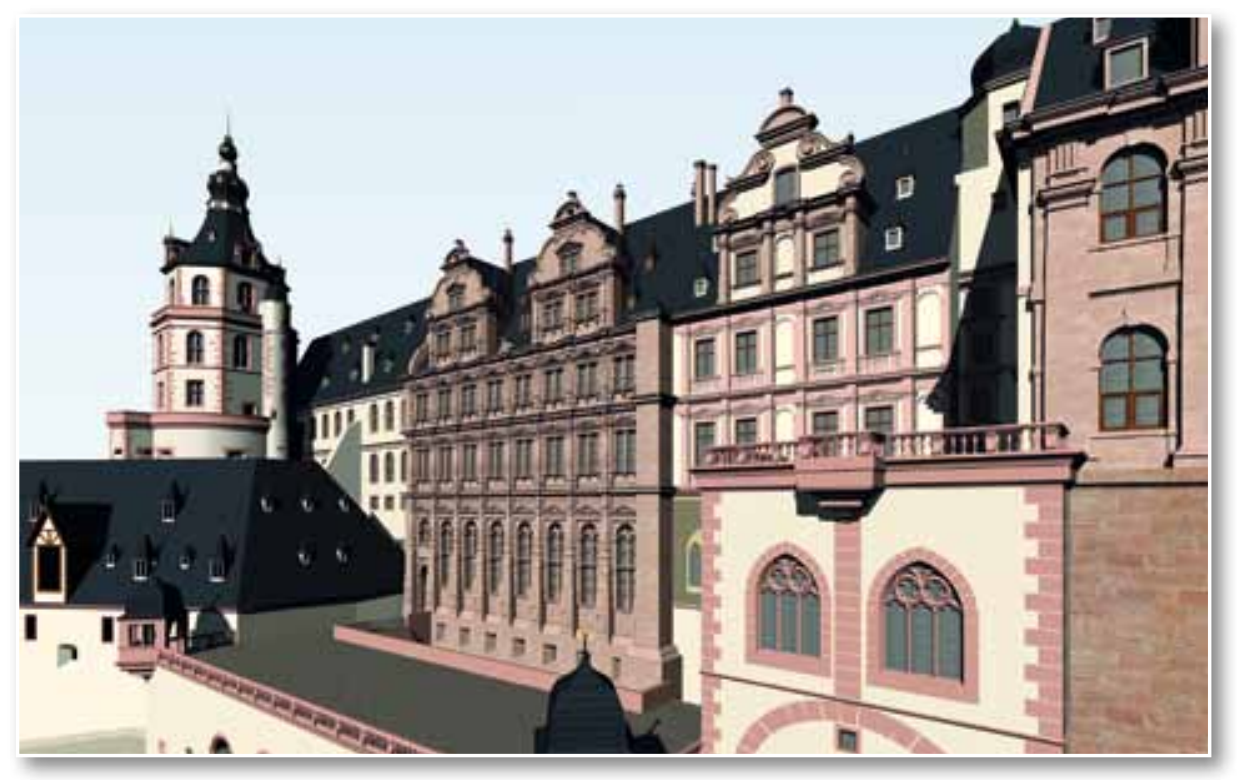

schließlich beim letzten Schlossbrand infolge eines Blitzschlages im Jahre 1764 zugrunde ging. ${ }^{19}$ Seit dieser Zeit stellt der Glockenturm eine bis auf wenige Ausbesserungen unverändert belassene Ruine dar.

Mit seinen vielfältigen bauhistorischen Spuren schildert der Glockenturm dem heutigen Betrachter in geradezu exemplarischer Weise die von Zerstörung und Wiederaufbau geprägte Architektur des Heidelberger Schlosses. In seiner heutigen ruinösen Gestalt demonstriert das Bauwerk zudem besonders eindrucksvoll die jüngere Geschichte des Heidelberger Schlosses.
Ein weiterer Bau, welcher der Zeit Friedrichs II. zuzuweisen ist, stellt das Obergeschoss des Ludwigsbaues dar, welches nach Ausweis der Kraus'schen Kupferstiche des Schlosshofes bis zu seiner barocken Erneuerung mit dem oberen Ok- 


\section{Literatur}

Bischoff 1999

Bischoff, Franz: Burkhard Engelberg „der vilkunstreiche Architector und der Statt Augspurg Wercke Meister". Burkhard Engelberg und die süddeutsche Architektur um 1500. Anmerkungen zur sozialen Stellung und Arbeitsweise spätgotischer Steinmetzen und Werkmeister.

Augsburg 1999.

Ebel 1915

Ebel, Friedrich: Ersatz des am 29. März 1915 verbrannten Wetzlarer Skizzenbuches.

Frankfurt a. M. 1915.

Hohrath u.a. 1990

Hohrath, Daniel; Henning, Rudolf; Kieven, Elisabeth;

38 Schneider, Erich; Wenger, Michael:

Die Bildung des Offiziers in der Aufklärung.

Ferdinand Friedrich von Nicolai (1730-1814) und seine enzyklopädischen Sammlungen.

Stuttgart 1990.

Hubach 1996

Hubach, Hanns: Kurpfälzisches Skizzenbuch.

Ansichten Heidelbergs und der Kurpfalz um 1600.

Heidelberg 1996.

Hucek 1992

Hucek, Miroslav: Der Hradschin. Die Prager Burg und ihre Konzeption und Gestaltung.

Freiburg im Breisgau 1992.

Koch/Seitz 1891

Koch, Julius; Seitz, Fritz: Das Heidelberger Schloss. Darmstadt 1891.
Leodius 1624

Leodius, Hubertus Thomas: Annalium de vita et rebus gestis illustrissimi principis Friderici II. Electoris palatini, libri XIV.

Frankfurt 1624.

Metzger 1829

Metzger, Johann: Beschreibung des Heidelberger Schlosses und Gartens. Nach gründlichen

Untersuchungen und den vorzüglichsten

Nachrichten bearbeitet.

Heidelberg 1829.

Oechelhaeuser 1913

Oechelhaeuser, Adolf von: Die Kunstdenkmäler des Großherzogtums Baden. Beschreibende Statistik. Kreis Heidelberg. Die Kunstdenkmäler des Amtsbezirks Heidelberg.

Tübingen 1913.

Rosenberg 1882

Rosenberg, Marc: Quellen zur Geschichte des Heidelberger Schlosses.

Heidelberg 1882.

Seeliger-Zeiss 1967

Seeliger-Zeiss, Anneliese: Lorenz Lechler von Heidelberg und sein Umkreis. Studien zur Geschichte der spätgotischen Zierarchitektur und Skulptur in der Kurpfalz und in Schwaben.

Heidelberg 1967

(Heidelberger kunstgeschichtliche Abhandlungen; N. F., 10). 